\title{
New Modulation Zero-Shift Method to Characterize Fast Group Delay Ripple of Dispersion-Compensating Fiber Bragg Gratings
}

\author{
María C. Santos, Santa P. De Bernardo-Rodi, and Marco A. Mitre-Gutiérrez
}

\begin{abstract}
An upgrade to standard modulation phase-shift method is shown to improve accuracy through electrical scan of the optical spectrum. Experiments have allowed the capture of fast group delay ripple of a dispersion-compensating fiber Bragg grating with only a small modification to the standard setup.
\end{abstract}

Index Terms-Fiber Bragg gratings (FBGs), group delay ripple (GDR), optical fiber dispersion, optical fiber testing.

\section{INTRODUCTION}

D ISPERSION-COMPENSATING fiber Bragg gratings (DC-FBGs) are key to dispersion control in long-distance high-capacity optical links. Accurate characterization of their group delay spectrum is a critical issue especially in the evolution towards $40-\mathrm{Gb} / \mathrm{s}$ systems. A random ripple coming from imperfections and tolerances in the fabrication process has been reported featuring typical variations in the picometer and picosecond ranges. The impact of this group delay ripple (GDR) grows with the number of cascaded DC-FBG and depends on the GDR amplitude and frequency [1]-[3].

The modulation phase-shift method (MPSM) is well established as an all-purpose dispersion measurement and it gives the basis of compact laboratory equipment of increasingly extended use [4]. It produces excellent results in the characterization of long, broadband optical systems such as optical fibers. Interferometric methods are more convenient though, for characterizing narrowband dense wavelength-division-multiplexing devices [5].

The MPSM requires measurement of the electrical phase shift $\Delta \phi$, acquired by the envelope of a modulated optical carrier at frequency $f_{0}$ as it proceeds through the device under test (DUT), to approximate the group delay at $f_{0}$ as

$$
\tau_{g} \approx-\frac{\Delta \phi(\mathrm{rad})}{2 \pi f_{m}}
$$

where $f_{m}$ is the electrical frequency of the pure-sine modulating signal. It can be seen that $\Delta \phi$ is the semi-difference between the optical phase shifts at frequencies $f_{0}+f_{m}$ and $f_{0}-f_{m}$, i.e., the optical sidebands generated by the modulation, so that

Manuscript received March 5, 2007; revised May 24, 2007. This work was supported by the European Union (EU) FP6 NoE e-Photon/ONe, (JP-T workpackage) and the Spanish MEC Projects TEC2005-05160 and TEC2004-06194$\mathrm{C} 02$.

The authors are with the Optical Communications Group, Universitat Politècnica de Catalunya, Barcelona 08034, Spain (e-mail: santos@tsc.upc.edu).

Digital Object Identifier 10.1109/LPT.2007.902296

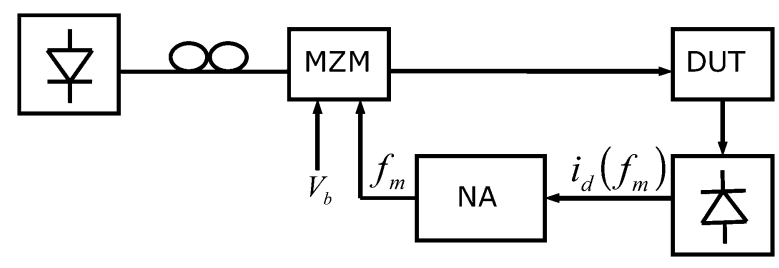

Fig. 1. Setup for the new MSZM.

(1) is a numerical approximation of the derivative of the phase shift against angular frequency. For characterizing fast variations in the group delay response, the sidebands should be close to the carrier, limited by the device-induced phase shift dropping below the measurement phase noise. The choice of $f_{m}$ is then subject to a trade-off between wavelength accuracy and amplitude uncertainty limiting the application of MPSM to the characterization of fast GDR [4], [6]. A further limitation comes from the stability and accuracy of the tunable laser used to scan the optical spectrum [7]. Electrical sweeps of the modulating frequency of a single-sideband modulated carrier have been proposed as a means to scan the optical frequency improving the accuracy of group delay measures [8].

In this letter, we present the basis of a new method that allows for electrical scan of the optical spectrum by using double-sideband modulation of the optical carrier. By addressing only one of the branches of a dual-drive optical modulator, the interference between optical sidebands is controlled through the bias voltage. A combination of the required bias to cancel detection with MPSM measures at two modulating frequencies yields on one side the phase-shift difference between the two upper sidebands as in [6] and on the other, also the phase-shift difference between the two lower sidebands, whereby group delays at both sides of the carrier can be calculated.

\section{THEORY}

The new modulation zero-shift method (MZSM) follows the scheme in Fig. 1. The optical carrier from a laser source is amplitude modulated in a dual-drive Mach-Zehnder modulator (MZM) asymmetrically driven, i.e., bias voltage and radio-frequency modulating signal are only applied to one of the interferometer branches. The normalized low-pass equivalent field at the MZM output in the small signal approximation is

$$
\bar{E}_{O}=\frac{1}{2}\left(1+e^{j\left(\theta_{b}+\theta_{m}\right)}\right) \approx \frac{1}{2}+\frac{e^{j \theta_{b}}}{2}\left(1+j \theta_{m}\right)
$$




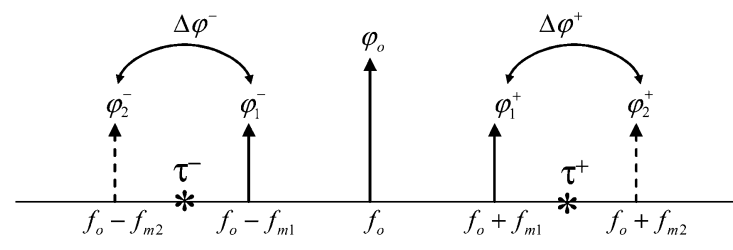

Fig. 2. Superposed spectra of the DUT outputs for two modulating frequencies, $f_{m 1}(-)$ and $f_{m 2}(--)$. The delta at $f_{o}$ is present into both signals. Stars: frequencies where group delays are calculated.

where $\theta_{b, m}=V_{b, m} \pi / V_{\pi} ; V_{b}, V_{\pi}$ are bias and half-wave voltages, respectively, and $V_{m}=A_{m} \cos \left(2 \pi f_{m} t\right)$ is the pure-sine modulating signal. It becomes evident from (2) that the optical sidebands are affected by a phase shift that depends on the bias voltage. That allows control of their mutual interference so that for each particular DUT, amplitude nulls occur at the desired $f_{m}$ frequency, adding radio-frequency accuracy to the fiber dispersion measurement technique in [9].

This signal is input to the DUT, a DC-FBG in this work, and gets detected at its output. For the normalized detected photocurrent at frequency $f_{m}$, we find

$$
\begin{aligned}
i_{d}\left(f_{m}\right)=-\frac{\alpha A_{m}}{2} & \cos \left(\frac{\theta_{b}}{2}\right) \sin \left(\frac{\theta_{b}}{2}+\Sigma \phi_{m}\right) \\
& \times \cos \left(2 \pi f_{m} t+\Delta \phi_{m}\right)
\end{aligned}
$$

where $\alpha$ is the radio-frequency modulation index accounting for all coupling and optical transmission losses. Information about the DUT phase-shift characteristic is contained into two magnitudes, namely $\Sigma \phi_{m}=\left(\varphi^{+}+\varphi^{-}-2 \varphi_{0}\right) / 2$ and $\Delta \phi_{m}=$ $\left(\varphi^{+}-\varphi^{-}\right) / 2$, with $\varphi_{0}, \varphi^{+}, \varphi^{-}$, the optical phase shifts added by the DUT at the optical carrier frequency, the upper and the lower sidebands, respectively.

As seen in (3), the photocurrent is taken to its minimum for bias voltages $V_{b f z}=(2 i+1) V_{\pi}$ and $V_{b m z}=2(i-\Sigma \phi / \pi) V_{\pi}$, with $i=0, \pm 1, \pm 2, \ldots$. The former nulls are "fixed" in the sense that they do not change their value as the modulating frequency is swept, while the latter do change because they depend on the DUT-induced phase shifts through the $\Sigma \phi$ quantity.

Group delay calculation requires finding the values of $V_{b m z 1}$ and $V_{b m z 2}$, and also those for $\Delta \phi_{m 1}, \Delta \phi_{m 2}$ corresponding respectively to two different modulating frequencies $f_{m 1}$ and $f_{m 2}$. The phase-shift differences between the respective two upper sidebands $\Delta \varphi^{+}=\varphi_{2}^{+}-\varphi_{1}^{+}$and that for the lower sidebands $\Delta \varphi^{-}=\varphi_{1}^{-}-\varphi_{2}^{-}$(see Fig. 2) are given by

$$
\Delta \varphi^{ \pm}=\Delta \phi_{m 2}-\Delta \phi_{m 1} \mp \frac{\pi}{2 V_{\pi}}\left(V_{b m z 2}-V_{b m z 1}\right) .
$$

Corresponding group delays at $f_{0}+\left(f_{m 1}+f_{m 2}\right) / 2, \tau_{g}^{+}$, and at $f_{0}-\left(f_{m 1}+f_{m 2}\right) / 2, \tau_{g}^{-}$, come from

$$
\tau_{g}^{ \pm} \approx-\frac{\Delta \varphi^{ \pm}}{2 \pi\left(f_{m 2}-f_{m 1}\right)} .
$$

Care has been taken when measuring the $\Delta \phi_{m i}$ because as the modulating frequency is increased, the DC-FBG-induced phase shifts may impact the detected photocurrent causing it to drop below required levels for correct phase determination if the bias

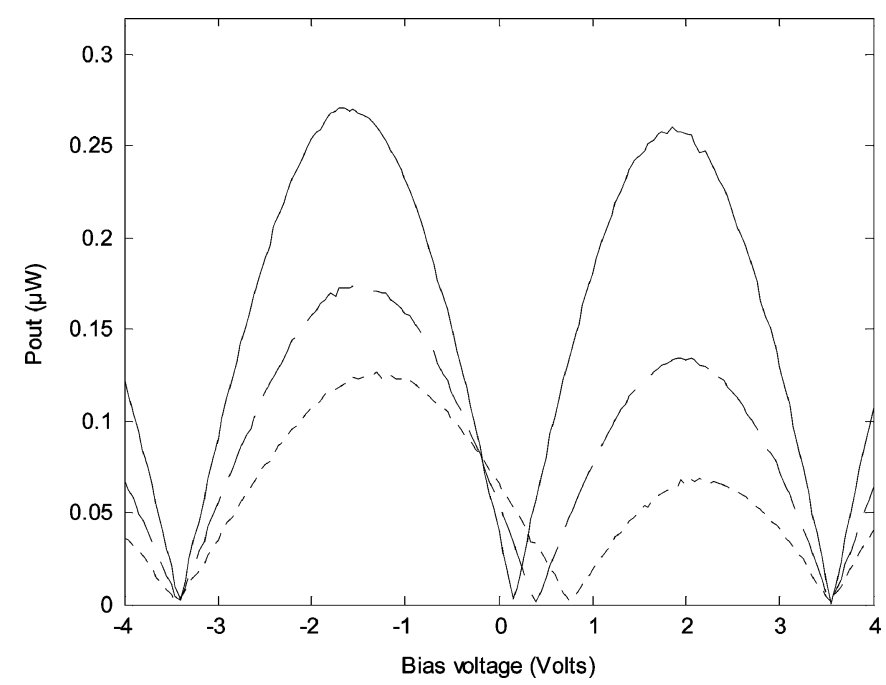

Fig. 3. Detected photocurrent against bias voltage for $1 \mathrm{GHz}$ (continuous line), $2 \mathrm{GHz}$ (dashed line), and $3 \mathrm{GHz}$ (dotted line).

voltage is kept at the quadrature point. In our experiments, the algorithm that obtains the voltage bias for minimum photocurrent also finds those best suited for phase measurements, i.e., the ones providing maximum detected photocurrent at $f_{m i}$.

\section{MEASUREMENTS}

\section{A. Characterization of Zero Amplitude Shifts}

As a first step towards MSZM implementation, we study here the characteristics of the zero detected amplitude shifts and their dependence with the bias voltage applied to only one of the arms of the MZM. Based on the scheme of Fig. 1, we have built an experimental setup which automatically implements the new MZSM through GPIB control. Detection and phase measurement take place at an HP 8703 A lightwave component analyzer (LCA) which also generates the modulating signal that is input to one of the arms of a dual-drive Fujitsu FTM7921ER/052 modulator with $V_{\pi}=3.55 \mathrm{~V}$ for the arm used. An HP 8168 A tunable laser with $-1-\mathrm{dBm}$ output power and wavelength fixed at $1559 \mathrm{~nm}$ goes through a polarizer optimized for maximum modulation efficiency. As a DUT, we have used a DC-FBG with 4- $\mathrm{nm}$ bandwidth centered at $1559 \mathrm{~nm}$ and nominal dispersion $-1252.35 \mathrm{ps} / \mathrm{nm}$.

In Fig. 3, we have plotted the amplitude of the detected photocurrent against bias voltage for three different modulating frequencies. We can distinguish there between the fixed zeros at odd multiples of $V_{\pi}$ and the moving zeros around even multiples of $V_{\pi}$. For the DC-FBG used here, the zero shift is on the order of $0.25 \mathrm{~V}$ for this range of frequencies. Since the phase-shift response for DC-FBGs basically scales as the square of the modulating frequency, so does the amplitude zero-shift because it is proportional to $\Sigma \phi$. Therefore, in order to reduce the measurement uncertainty, it is convenient to use high modulating frequencies. This is also true for the phase measurement. Since the frequency resolution is set by the $f_{m}$ frequency step rather than by the spacing between sidebands and carrier, the wavelength 


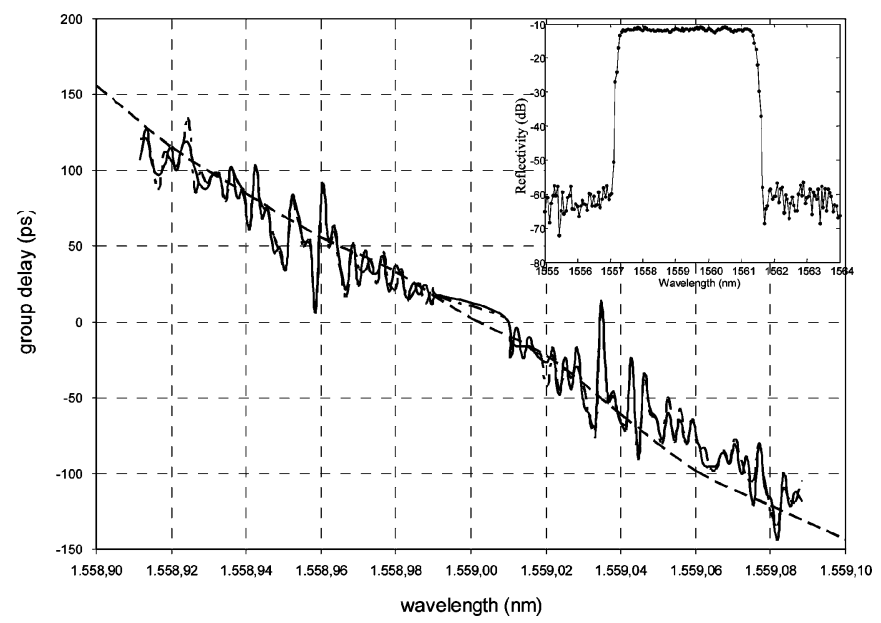

Fig. 4. Group delay spectra. MZSM $\Delta f=200 \mathrm{MHz},(-)$ and (-.); MPSM $(--) f_{m}=200 \mathrm{MHz}, \Delta \lambda=20 \mathrm{pm}$. Inset: reflectivity spectrum.

accuracy is maintained and the group delay amplitude uncertainty is reduced as in [6].

\section{B. MZSM Results}

In the MZSM automatic implementation, we have used frequencies from $130 \mathrm{MHz}$ to $11 \mathrm{GHz}$ in $\Delta f=200 \mathrm{MHz}$ steps and we have stored the LCA modulating frequency sweeps for bias voltages going from -4 to $4 \mathrm{~V}$ in $10-\mathrm{mV}$ steps. Results are shown in Fig. 4 together with MPSM results with a 200-MHz modulating frequency and $\Delta \lambda=20 \mathrm{pm}$ tunable laser step for comparison. We have checked that this is the smallest stepsize for our laser to give accurate and stable sweeps [7]. Consistent results in two different realizations of the MZSM experiment reveal reduced impact of measurement noise and prove that by extending the frequency range of the required phase measurement, the MZSM is able to resolve the DC-FBG oscillatory behavior smoothed out when using MPSM due to low stability and accuracy of our laser. We have obtained a ripple of about 3-pm period and 20-ps amplitude. When comparing these results to those in [4], we see that we have found similar values of GDR amplitude but we have been able to resolve an almost four times smaller period.

Because of increasing complexity of radio-frequency hardware as the modulating frequency grows, the total optical spectrum that can be electrically swept is limited. Thus, in practice in order to cover the overall operative DC-FBG bandwidth, the MZSM process will need to be repeated with a new optical carrier. The stepsize requirements of the tunable laser source will be significantly relaxed, though. A combination of optical and electrical frequency sweeps is envisioned where for each optical carrier tightly spaced high modulating frequencies are used so that larger zero shifts and phase differences to be measured help

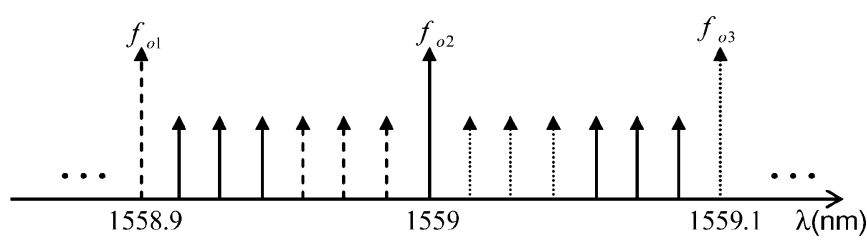

Fig. 5. Combination of electrical $(6-12 \mathrm{GHz})$ and optical $(\Delta \lambda=100 \mathrm{pm})$ frequency sweeps. Same style arrows are superposed spectra of signals in the electrical sweep using optical carrier $f_{o i}$.

to reduce the impact of measurement noise (Fig. 5). As an example, electrical scans from 6 to $12 \mathrm{GHz}$ could be combined with a $100-$ pm optical scan.

\section{CONCLUSION}

We have demonstrated a new optical group delay measurement method based on standard MPSM which by electrical scan of the optical spectrum allows for improved accuracy levels with relaxed requirements over the tunable source. Measures over a DC-FBG have yielded well-defined ripples of about 3-pm period and 20-ps amplitude. With little modifications to existing test equipment, the techniques described should allow us to measure the group delay characteristics of optical components to the detail demanded by long-distance high-data-rate applications.

\section{ACKNOWLEDGMENT}

The authors would like to thank Prof. J. Prat for helpful discussions and Dr. V. Polo for assistance in the experiments.

\section{REFERENCES}

[1] K. Ennser, M. Ibsen, M. Durkin, M. N. Zervas, and R. I. Laming, "Influence of nonideal chirped fiber grating characteristics on dispersion cancellation," IEEE Photon. Technol. Lett., vol. 10, no. 10, pp. 1476-1478, Oct. 1998.

[2] C. Scheerer et al., "Influence of filter group delay ripples on system performance," in Proc. ECOC, 1999, pp. 1410-1411.

[3] L.-S. Yan et al., "Investigation of performance variations due to the amplitude of group-delay ripple in chirped fibre Bragg gratings," Opt. Fiber Technol., vol. 12, pp. 238-242, Feb. 2006.

[4] R. Fortenberry, W. V. Sorin, and P. Hernday, "Improvement of group delay measurement accuracy using a two-frequency modulation phaseshift method," IEEE Photon. Technol. Lett., vol. 15, no. 5, pp. 736-738, May 2003.

[5] M. Froggatt et al., "Interferometric measurement of dispersion in optical components," in Proc. OFC, 2002, pp. 252-253.

[6] R. M. Fortenberry, "Enhanced wavelength resolution chromatic dispersion measurements using fixed sideband technique," in Proc. OFC, 2002, pp. 107-109.

[7] M. Soler, J. Prat, and M. C. Santos, "Error sources of chromatic dispersion measurement using dispersion compensating grating," in IEEE/LEOS Workshop Fiber and Optical Passive Components, Glasgow, U.K., Jun. 2002, pp. 213-218.

[8] J. E. Román et al., "Spectral characterization of fiber gratings with high resolution," Opt. Lett., vol. 23, pp. 939-941, 1998.

[9] C. Peucheret, F. Liu, and R. J. S. Pedersen, "Measurement of small dispersion values in optical components," Electron. Lett., vol. 35, pp. 409-411, Mar. 1999. 